\title{
Evaluation of an Enhanced Multicast Data Flow Technique in Network Mobility
}

\author{
Azana Hafizah Mohd Aman ${ }^{1}$, Aisha-Hassan A. Hashim ${ }^{1}$, Azween Abdullah ${ }^{2}$, \\ Huda Adibah Mohd Ramli ${ }^{1}$ and Shayla Islam ${ }^{1}$ \\ ${ }^{1}$ Kulliyyah of Engineering, International Islamic University Malaysia, Jln \\ Gombak 53100, Kuala Lumpur, Malaysia \\ ${ }^{2}$ SOCIT, Taylor's University, Jalan Taylors, Subang Jaya 47500, Selangor, \\ Malaysia
}

\begin{abstract}
Mobile IPTV applications are becoming very popular worldwide. According to CISCO research, mobile Internet video usage is increasing rapidly each year and soon reaching zettabyte threshold in 2019. With the increase number of traffic, it is a great effort for internet service provider to provide acceptable network performance. The objective of this paper is to mathematically evaluate a new proposed mobile multicast technique to support high performance mobile IP traffic. The new technique enhanced multicast services in Network Mobility Management which is Proxy Mobile IPv6 (PMIPv6). The evaluation is done using mathematical equations that are derived from signaling call flow of the multicast process in the proposed technique. Results are analyzed and compared with the current base solution technique. Using mathematical analysis this paper validates and highlights the strengths of the proposed technique compared to the existing technique.
\end{abstract}

Keywords: Multicast, Mobile, PMIPv6, Mobility Management

\section{Introduction}

According to CISCO recent white paper [1-3], yearly worldwide IP traffic will exceed the two zettabyte threshold in 2019. Over half of all IP traffic will come from non-PC devices such as mobile devices. Globally, internet video traffic will be 80 percent of all Internet traffic in 2019. So thus mobile data traffic that will grow three times faster than fixed IP traffic from 2014 to 2019. Mobile includes mobile data and Internet traffic generated by handsets, notebook cards, and mobile broadband gateways [1-3]. Internet video includes short-form and long-form Internet video, live Internet video, Internetvideo-to-TV, online video purchases and rentals, webcam viewing, and web-based video monitoring [1-3]. Although multicast mobility has been a concern for about more than ten years and has led to numerous proposals, there is as yet no generally accepted solution [45].

Mobility in IPv6 is standardized in the Mobile IPv6 [6], and it is the concept of network-layer changes while moving between wireless network. MIPv6 [6] roughly defines multicast mobility for Mobile Nodes (MNs) using a remote subscription approach or through bidirectional tunneling via the Home Agent (HA). Remote subscription suffers from slow handovers due to multicast routing [4-5]. Bidirectional tunneling introduces inefficient overhead and delay due to triangular forwarding [4-5]. As a solution to this issues and the huge increase in mobile multicast IP traffic, this research proposed an enhancement to support high data delivery performance in Mobile IPv6 (MIPv6) [6]. The enhanced technique is based on multicast method with Proxy Mobile Ipv6 (PMIPv6) [7-8]. In the proposed technique the multicast traffic is processed and 
delivered using the integration of Route Optimized [9- 11] Context Transfer [12-14] named as RC and Multicast Fast Reroute [15] named as MFR scheme. The new enhanced technique is named as RCMFR. This integration improves handover performance. As well as the process of delivering the required multicast information is shortened. This paper evaluates the proposed enhancement using quantitative approach. The proposed enhancement is benchmarked with the standard base technique [16] of PMIPv6 (proposed by IETF). The mathematical analyses are evaluated based on the selected parameters which are the total number of signals and, handover latency. All parameters are derived from the handover process flow.

This paper is organized as follows. Section 2 describes related work, then section 3 the proposed enhancement technique. This is followed by the quantitative analysis in section 4. The results and discussion of the selected solution is elaborated in Section 5. Finally, the conclusion of the paper is presented in Section 6.

\section{Related Work}

Current related works on multicast PMIPv6 techniques are route optimization [20-22], global mobility [23], load balancing [24] and context transfer [25-27].

[20-22] use route optimization technique. Introduces an effective handover technique for multicast to provide low handover delay and packet loss. Provide shorter service disruption because it joins the multicast group in advance with no binding update and multicast tree joins delay. Traffics are buffered at the destination location during a handover. The suggested technique beats existing base multicast scheme handover performance for end-to-end delay, the amount of lost packets and session disruption time. But this technique lack of inter-domain mobile node handover and the signaling cost of the operations are missing. Hence enhancement is required. [23] provides inter domain multicast mobility. It overcome the base, dedicated and direct routing techniques that only preserve the intra domain mobile node movement. It maintains the global mobility movement by using extra signaling between LMAs. The signal is called Session Mobility Anchor (SMA). This technique is lack of intra domain support and the extra signals resulted in high signaling cost.

[24] solves traffic issue at the LMAs. The LMA is liable for retaining the mobile node's accessibility state. This is also responsible for sending the data traffic from and to the present position of the MN in PMIPv6. As mobile multicast traffic quickly growing, it clearly creates a blockage as well as a single point of failure at the LMA. The solution supports to better allocate the load between the LMAs while decreasing the multicast support interruption. The solution balances the multicast load among LMAs by using Load Balancing Controller (LBC). This solution only considers inter domain and lack of parameters performance evaluations. [25-27] describe techniques using Context Transfer Protocol. The aim is to provide continuous multicast service for PMIPv6. When a multicast mobile node disconnects from the existing MAG and connects to a new MAG, the node is capable to get the multicast data instantly from the new MAG. Hence provides multicast session continuity with lesser packet loss. However these works only consider intra domain interchange and lack of performance validation.

\section{Proposed Enhancement}

This paper focused on the predictive method of context transfer in RCMFR. It is a new enhancement to the current PMIPv6, by combining MFR with RC. This combination enhances the handover performance in proxy mobile multicast environment. Multicast information is used in PMIPv6 to build multicast distribution trees by multicast routing protocols [17]. This multicast distribution trees are used to serve the listeners in a network. 
The design minimizes the time needed to re-establish the service as much as possible. As well as minimize the number of signaling for the control and join messages. The design assumes that all connected routers are multicast in PMIPv6 (MPMIPv6) environment. It assumes the presence of multicast Local Mobility Anchor (LMA) and multicast Mobile Access Gateway (MAG). This MAG is responsible for controlling the group membership for multicast listeners (MLDv2) [17] and receives the multicast packets intended to the Mobile Node (MN) then encapsulates them to the MN. It is assumed that the transfer for intra domain is localized between two MAGs to ensure efficient MLDv2 [17] context transfer based on minimal communication. It is assumed that pMAG and nMAG is the Provider Edge (PE) [15] for the MN multicast group communication. As the PEs of the RCMFR both pMAG and nMAG received multicast data simultaneously from the LMA. In RCMFR, the LMA is the router that serves as multicast subscription anchor point, while the MAG provides MLDv2 proxy functions. All multicast is directed from LMA to MAG. This traffic direction is done using Proxy Care of Address (PCoA) as an identifier with respect to the multicast routing tree. The context transfer is used during a mobile node's handover in order to provide the next MAG with the mobile node's listening state. This is important so that the various routing contexts are established on behalf of the mobile node. It is assumed that all the scenarios described in this paper, is supported by the RC and MFR. In order to deliver the packets to the mobile node with better handover performance.

The network model for the proposed technique for intra domain and inter domain handover is shown in Figure 1. As for this paper the analysis is only done for intra domain multicast communication. The inter domain multicast communication analysis is considered as future work. As shown in the figure, the network contains three levels, the highest level is where Local Mobility Anchor (LMA) resides, the second level is the location of Mobile Access Gateway (MAG) and the lowest level is the location of the Mobile Node (MN). The implementation of the MFR and RC operations is at the LMAs and MAGs. Figure 2 and Figure 3 shows the handover signaling flow for the MPMIPv6 $[7,8]$ and the MPMIPv6 with RC respectively. The signaling flow of the proposed scheme MPMIPv6 RCMFR is shown in Figure 4.
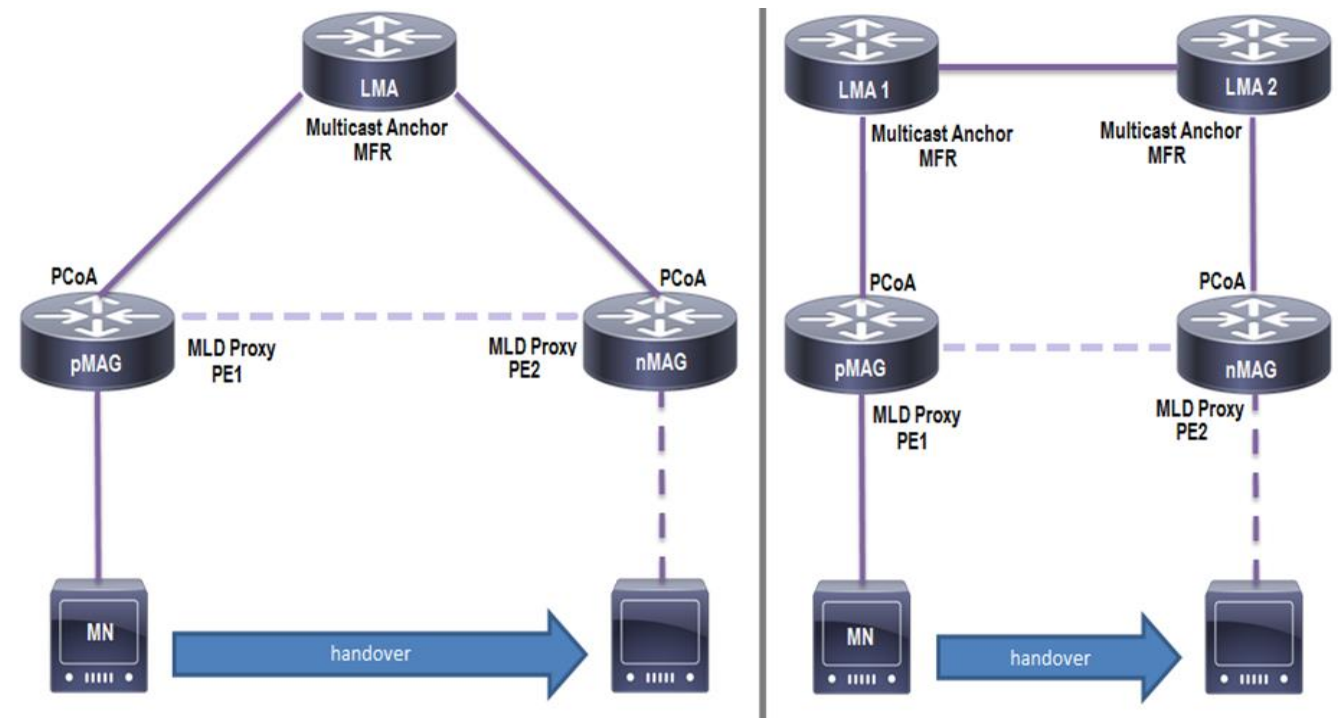

Figure 1. Network Model for the proposed Technique 


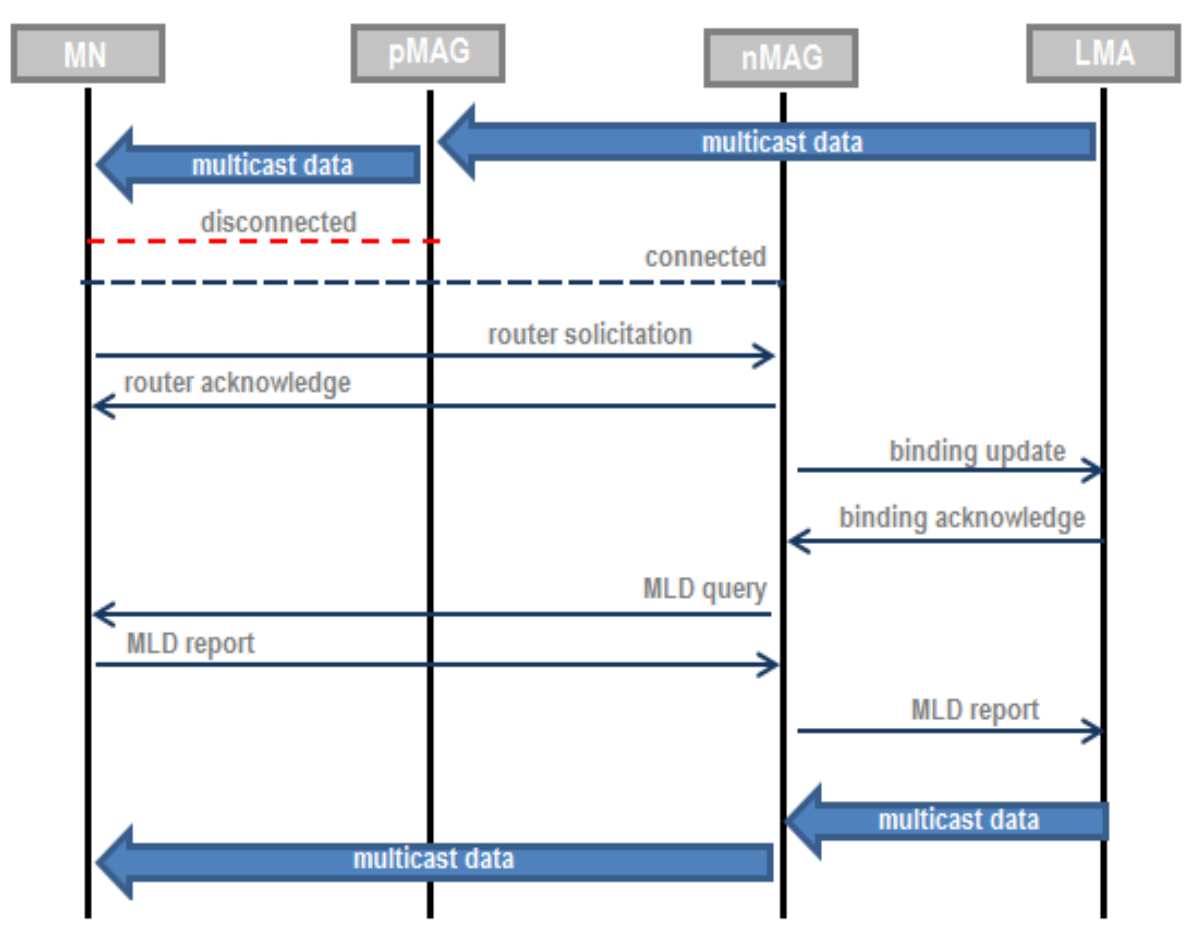

Figure 2. MPMIPv6 Handover Signaling Flow [7-8]

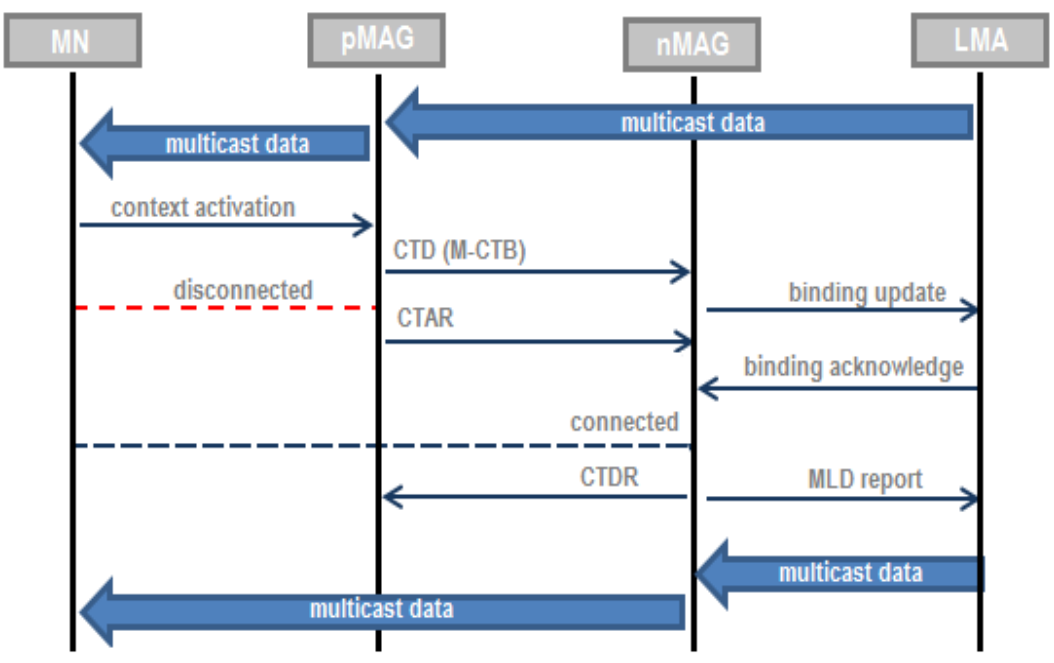

Figure 3. MPMIPv6 with RC Handover Signaling Flow 


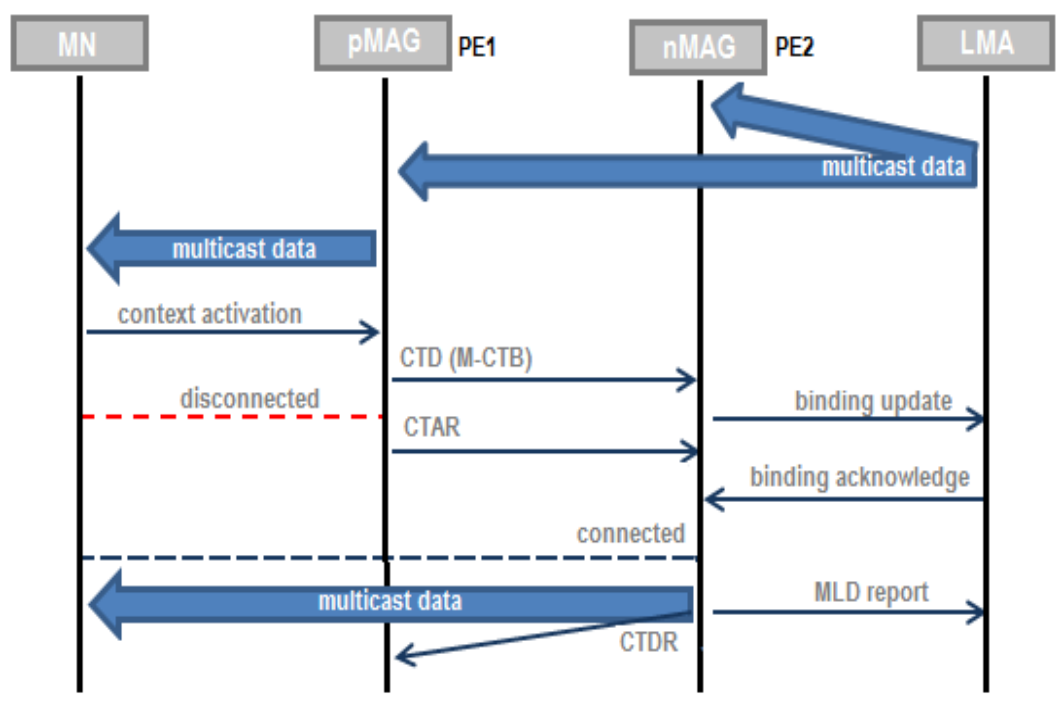

Figure 4. MPMIPv6 RCMFR Handover Signaling Flow

At the initial state of handover process, the MN sends context activation to the pMAG. The multicast context transfer block (M-CTB) is built at pMAG. The M-CTB includes the multicast addresses required for the multicast services being used by the moving mobile node. This block is transferred to the nMAG by pMAG. With the implementation of MFR at the MAGs, once the MN moves to the nMAG, the MN will be able to receive the multicast packets immediately. This is because the nMAG is the PE member of the multicast group. As for the LMA update, upon connection to nMAG it has already sent the join update to the LMA. Then the MLDv2 supplies the information the multicast routing protocol to build the routing context for the multicast addresses.

Upon receiving the context data message the nMAG dispatches it to the MLDv2 router. This is only to update the aggregate state. As the individual node context is already established since it is the PE. A change in the aggregated state will then trigger the active multicast routing protocol to build the distribution trees for each of the multicast groups. In the RC handover process, pMAG has to send a Context Transfer Active Request (CTAR) message to inform MN previous location. The CTAR message is sent to the nMAG, where the mobile node is connected. The CTAR includes the pMAG's IP address. The Context Transfer Data Reply (CTDR) message is sent by nMAG to pMAG to indicate the success or failure of context transfer.

Through this implementation, the time needed to re-establish the service is reduced. This is due to the fact that the multicast information is transferred between the two MAGs before the handover is completed. Plus the nMAG is one of the PE of the MN for the multicast group. Hence all the information needed for the MN to join the multicast group is already transferred. The $\mathrm{MN}$ can received the data of the multicast group as soon as the MN moves to the nMAG. The number of signaling involve after the handover is also reduced since the communication is localized between the two MAGs. The mobile node doesn't involve in sending the group membership message to the nMAG or LMA.

\section{Quantitative Analysis}

The mathematical equation for the total number of signaling, and handover latency is derived from the handover signaling flow in section 2.

\subsection{Total Number of Signaling}

The total number of signaling is described as all signaling messages involved in the transfer of the MN from previous Mobile Access Gateway (pMAG) to new Mobile 
Access Gateway (nMAG). Let $\alpha$ s denoted as total number of signaling. The other factor involved is the number of multicast group of the MN. Let $n$ denoted as the number of multicast group. Therefore the total number of signaling for each solution is defined as below:

The total number of signaling for a multicast group in MPMIPv6, $\alpha_{\mathrm{s}-\mathrm{MPMIPv} 6}$ is;

$$
\alpha_{\mathrm{s}-\mathrm{MPMIPv} 6}=S_{R S}+S_{R A}+S_{B U}+S_{B A}+S_{M L D q}+S_{M L D r 1}+S_{M L D r 2+} S_{M D 1}+S_{M D 2}
$$

While for $n$ number of multicast group for a MN in MPMIPv6,

$$
\alpha_{\mathrm{s}-\mathrm{MPMIPV} 6} \text { is }=n \cdot \alpha_{\mathrm{s}-\mathrm{MPMIPv} 6}
$$

Where Table 1 summarizes the parameters for $\alpha_{\mathrm{s}-\mathrm{MPMIPv} 6}$ evaluation;

Table 1. Parameters for $\alpha_{\text {s-MPMIPv6 }}$

\begin{tabular}{ll}
\hline Notation & Description \\
\hline$S_{R S}$ & Router Solicitation message from MN to nMAG \\
$S_{R A}$ & Router Acknowledgement message from nMAG to MN \\
$S_{B U}$ & Binding Update message from nMAG to LMA \\
$S_{B A}$ & Binding Acknowledge message from LMA to nMAG \\
$S_{M L D q}$ & MLD query message from nMAG to MN \\
$S_{M L D r 1}$ & MLD report message from MN to nMAG \\
$S_{M L D r 2}$ & MLD report message from nMAG to LMA \\
$S_{M D 1}$ & Multicast Data message from LMA to nMAG \\
$S_{M D 2}$ & Multicast Data message from nMAG to MN \\
\hline
\end{tabular}

However in case of MPMIPv6 with RC, the total number of signaling is denoted as $\alpha_{\mathrm{s}-\mathrm{RCMPMIPv}}$ therefore;

$$
\alpha_{\mathrm{s}-\mathrm{RCMPMIPv} 6}=S_{C A}+S_{C T D}+S_{C T A R}+S_{B U}+S_{B A}+S_{C T D R}+S_{M L D r+} S_{M D I}+S_{M D 2}
$$

While for $n$ number of multicast group for a MN in MPMIPv6 with RC, $\alpha_{\mathrm{s}-\mathrm{RCMPMIPv} 6}$ is ;

$$
=n \cdot \alpha_{\mathrm{s}-\mathrm{RCMPMIPv} 6}
$$

Where Table 2 summarizes the parameters for $\alpha_{s-R C M P M I P v 6}$ evaluation;

\section{Table 2. Parameters for $\boldsymbol{\alpha}_{\text {s-RCMPMIPv6 }}$}

\begin{tabular}{ll}
\hline Notation & Description \\
\hline$S_{C A}$ & Context Activation message from MN to nMAG \\
$S_{C T D}$ & Context Transfer Data message from pMAG to nMAG \\
$S_{C T A R}$ & Context Transfer Active Request message \\
$S_{B U}$ & Binding Update message from nMAG to LMA \\
$S_{B A}$ & Binding Acknowledge message from LMA to nMAG \\
$S_{C T D R}$ & Context Transfer Data Reply message from nMAG to pMAG \\
$S_{M L D r}$ & the MLD report message from nMAG to LMA \\
$S_{M D 1}$ & Multicast Data message from LMA to nMAG \\
$S_{M D 2}$ & Multicast Data message from nMAG to MN \\
\hline
\end{tabular}

As for the proposed enhancement MPMIPv6 with RCMFR, the total number of signaling is denoted as $\alpha_{\mathrm{s}-\mathrm{RCMFR} / \mathrm{MPMIPv} 6}$ therefore;

$$
\alpha_{\mathrm{s}-\mathrm{RCMFR} / \mathrm{MPMIPv} 6}=S_{C A}+S_{C T D}+S_{C T A R}+S_{B U}+S_{B A}+S_{C T D R}+S_{M L D r+} S_{M D 1}
$$


While for $n$ number of multicast group for a MN in MPMIPv6 with RCMFR, $\alpha_{s-}$ RCMFR/MPMIPv6 is;

$$
=n \alpha_{\mathrm{s}-\mathrm{RCMFR} / \mathrm{MPMIPv} 6}
$$

Where Table 3 summarizes the parameters for $\alpha_{\text {s-RCMFR/MPMIPv6 }}$ evaluation;

Table 3. Parameters for $\alpha_{\text {s-RCMFR/MPMIPv6 }}$

\begin{tabular}{ll}
\hline Notation & Description \\
\hline$S_{C A}$ & the Context Activation message from MN to nMAG \\
$S_{C T D}$ & the Context Transfer Data message from pMAG to nMAG \\
$S_{C T A R}$ & the Context Transfer Active Request message from pMAG to \\
& nMAG \\
$S_{B U}$ & the Binding Update message from nMAG to LMA \\
$S_{B A}$ & the Binding Acknowledge message from LMA to nMAG \\
$S_{C T D R}$ & the Context Transfer Data Reply message from nMAG to pMAG \\
$S_{M L D r}$ & the MLD report message from nMAG to LMA \\
$S_{M D 1}$ & the Multicast Data message from LMA to nMAG \\
\hline
\end{tabular}

\subsection{Handover Latency}

The handover latency is defined as the time needed for the MN to change its point of attachment from one network connection to another [18,19]. As in PMIPv6 network it is the time interval from the time MN disconnected from pMAG until the time MN connected to nMAG and received the first data packet from nMAG. It consist of router discovery delay and also link switching delay [18]. Let $\beta_{h}$ denoted as handover latency, $I_{l s}$ as link switching delay, $I_{R D}$ denoted as router discovery delay. Therefore the handover latency for each solution is defined as below:

Therefore the handover latency for MPMIPv6, $\beta_{h \text {-MPMIPv6 }}$ is:

$$
\beta_{h-\mathrm{MPMIPv} 6}=I_{l s}+I_{R D}+4 I_{M N n M A G}+3 I_{n M A G L M A}
$$

However in case of MPMIPv6 with RC, the handover latency, $\gamma_{h \text {-RCMPMIPv6 }}$

$$
\beta_{h-\mathrm{RCMPMIPv} 6}=I_{l s}+2 I_{\mathrm{pMAGnMAG}}+3 I_{\mathrm{nMAGLMA}}
$$

As for the proposed enhancement MPMIPv6 with RCMFR, the handover latency, $\gamma_{h}$. RCMFR/MPMIPv6

$$
\beta_{h-\mathrm{RCMFR} / \mathrm{MPMIPv} 6}=I_{l s}+I_{p M A G n M A G}+I_{L M A n M A G}
$$

Where Table 4 summarizes the parameters for $\gamma_{h \text {-MPMIPv6 }}, \gamma_{h \text {-RCMPMIPv6 }}$, and $\gamma_{h}$ RCMFR/MPMIPv6 evaluation;

Table 4: Parameters for Handover Latency

\begin{tabular}{ll}
\hline Parameter & Description \\
\hline$I_{p M A G n M A G}$ & time interval between pMAG and nMAG \\
$I_{\text {LMAnMAG }}$ & time interval between LMA and nMAG \\
$I_{M N n M A G}$ & time interval between MN and nMAG \\
$I_{\text {IMAGLMA }}$ & time interval between nMAG and LMA \\
$I_{\mathrm{nMAGLMA}}$ & time interval between LMA and nMAG \\
\hline
\end{tabular}




\section{Results and Discussion}

The numerical results based on the derivations in Eq. 1 to Eq. 9 is presented here. In ensuring a level comparative platform, the assumptions used by $[18,19]$ have been used in the research. They are as follows:

\subsection{Total Number of Signaling}

Figure 5 shows the total number of signaling versus number of multicast group. As shown the total number of signal increases as the number of multicast group increases. The increament for the proposed technique is the lowest than the other two techniques.

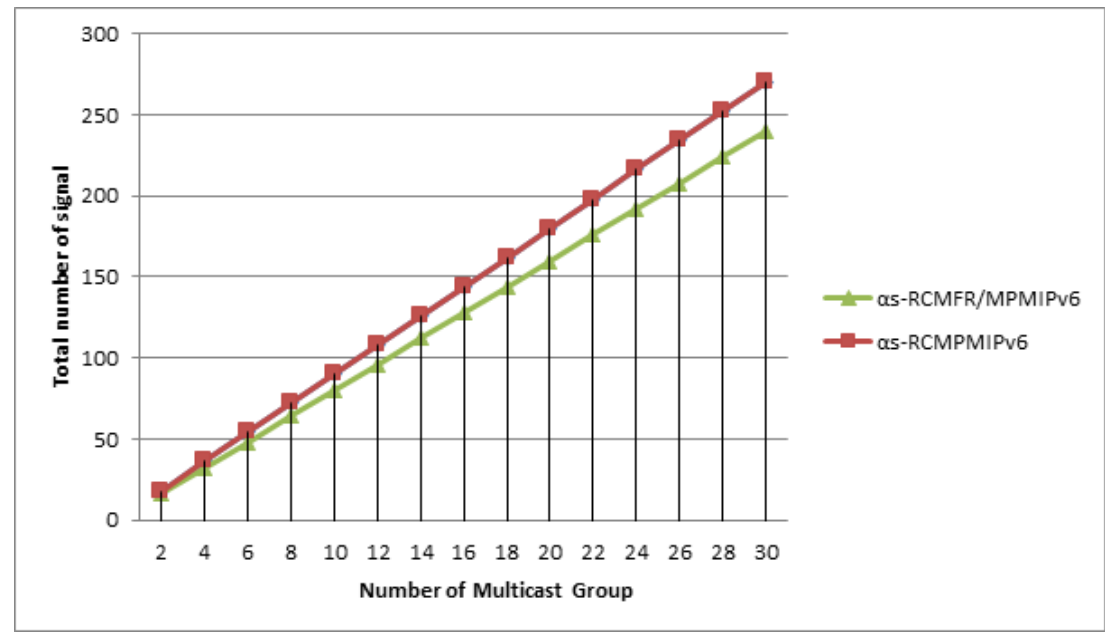

Figure 5. The Total Number of Signaling versus Number of Multicast Group

\subsection{Handover Latency}

Figure 6 shows handover latency for all the techniques. As shown the handover latency for the proposed technique outcome the other two techniques.

In comparison of link switching delay as shown in Figure 7, the handover latency increases as the link switching delay increases. However the proposed technique still has the lower handover latency compared to the other two techniques. As for handover latency versus router discovery as shown in Figure 7, the MPMIPv6 handover latency increases as the link switching delay increases. However for RCMPMIPv6 and the proposed technique both remain consistant. Still the proposed technique has the lower handover latency compared to the other two techniques. 


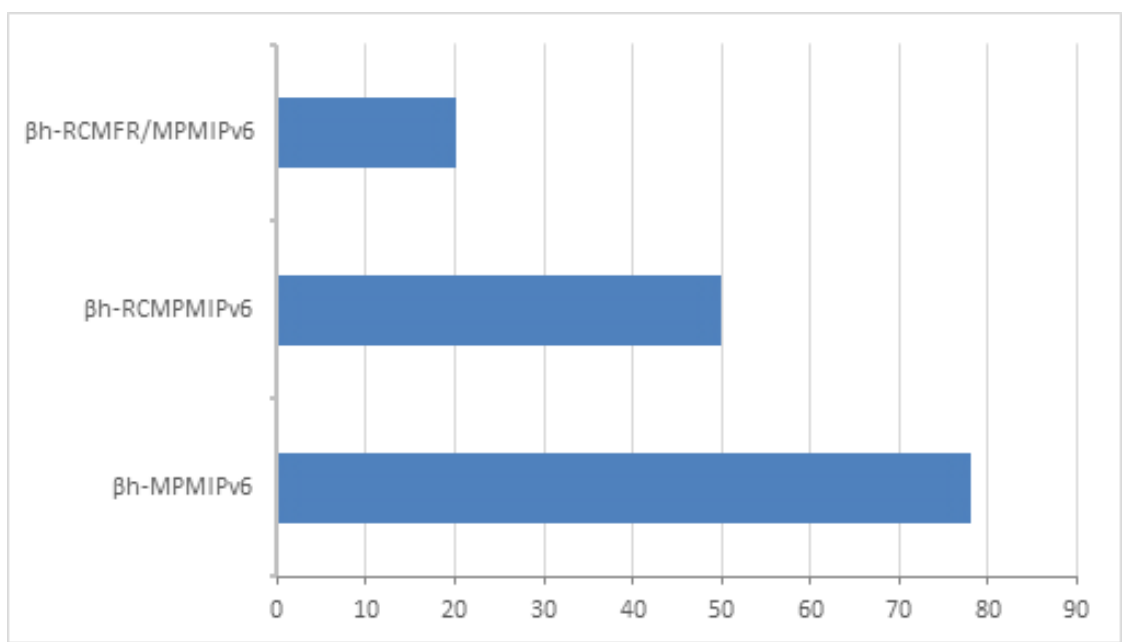

Figure 6. Handover Latency

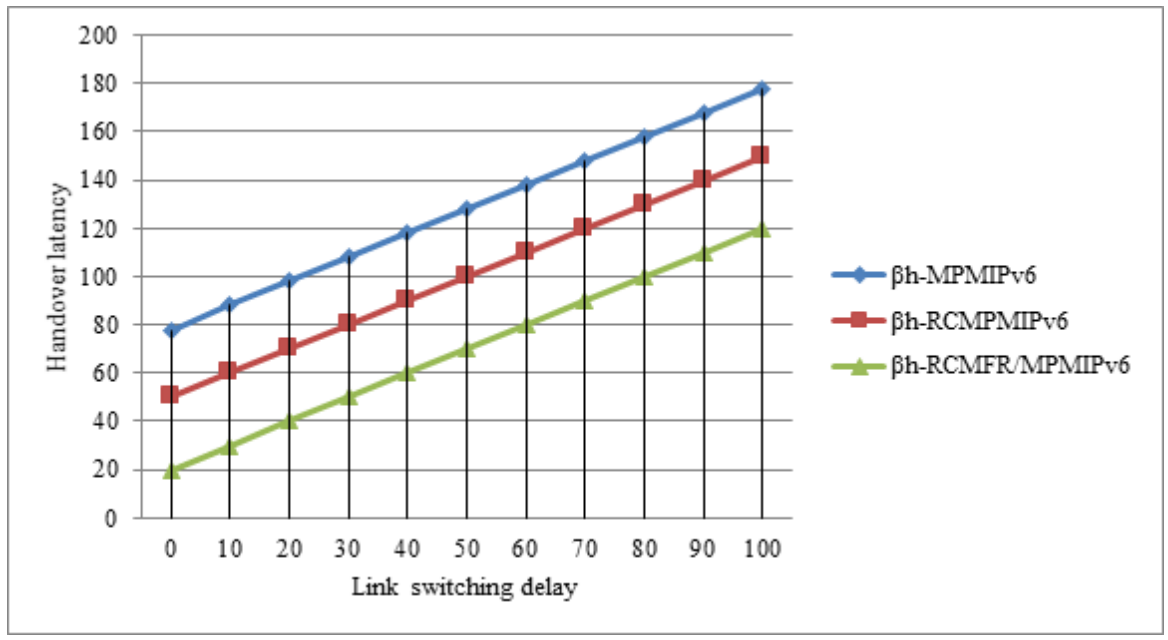

Figure 7 Handover Latency versus Link Switching Delay

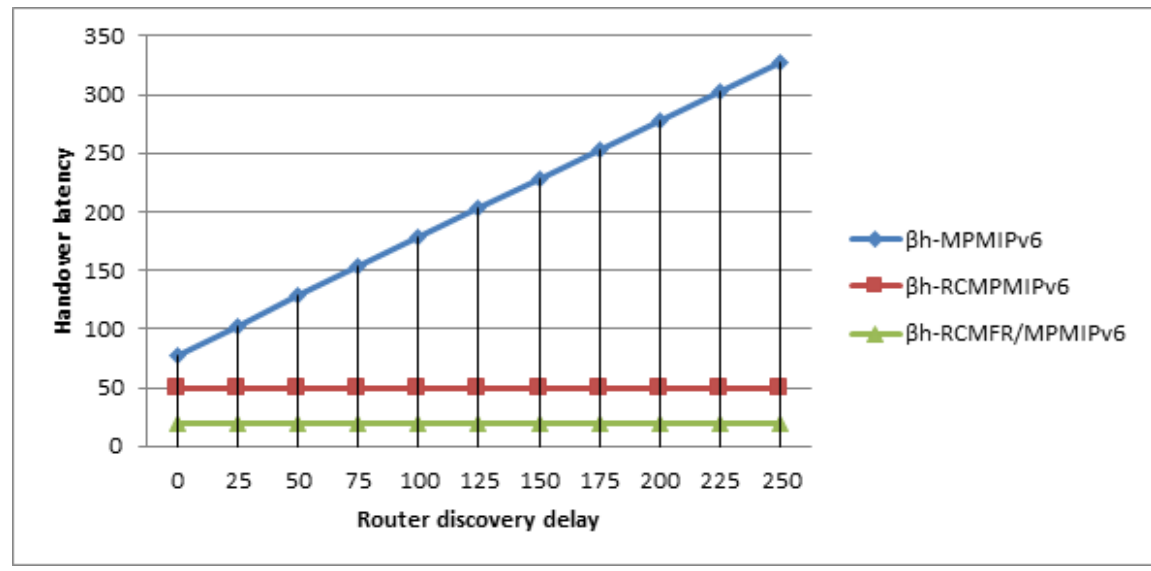

Figure 8. Handover Latency versus Router Discovery Delay 


\section{Conclusion}

This paper mathematically evaluates a new proposed mobile multicast technique to support high performance mobile IP traffic. The technique enhanced multicast support or services in Network Mobility Management which is Proxy Mobile IPv6 (PMIPv6). The mathematical equations are derived from signaling flow of the multicast process in the proposed technique. Results are analyzed and compared with the current base solution technique. From the equations and results, the proposed technique provides a better handover for mobile traffic. In conclusion, the combination of MFR and RC, the enhanced RCMFR technique reduces the service interruption of the basic PMIPv6. Compared to PMIPv6, the enhanced technique offers considerable total number of signal flow since the number of signaling involved is smaller than PMIPv6. Besides that the enhanced technique can shorten the handover latency time for multicast traffic. Therefor it is proven that by applying the enhanced scheme, the multicast data flow is more efficient than PMIPv6 and costs less performance degradation compared to the PMIPv6 scheme. In order to understand the applicability and efficiency of the proposed technique, experimental evaluations using network simulator are considered as future recommendation.

\section{References}

[1] Cisco Visual Networking Index: Global Mobile Data Traffic Forecast Update 2014 - 2019, Cisco White Paper, (2015).

[2] Cisco Visual Networking Index: Forecast and Methodology 2014 - 2019, Cisco White Paper, (2015).

[3] Cisco Visual Networking Index: The Zettabyte Era Trends and Analysis, Cisco White Paper, (2015).

[4] T. C. Schmidt, M. Waehlisch and G Fairhurst, "Multicast mobility in MIPv6: problem statement \& brief survey," RFC 5757, (2010).

[5] C. J Bernardos, M. Calderon and I. Soto, "PMIPv6 and network mobility problem statement," InternetDraft, (2012).

[6] D. Johnson, C. Perkins and J. Arkko, "Mobility support in IPv6", RFC 3775, (2004).

[7] S. Gundavelli, E. Leung, K. Devarapalli, V. K. Chowdhury and B. Patil, "Proxy Mobile IPv6(PMIPv6)", RFC 5213, (2008).

[8] D. Kim, W. S. Lim and Y. J. Suh, "Multicast extension to Proxy Mobile IPv6 for mobile multicast services", Journal of Computing Science and Engineering, vol. 5, (2011), pp. 316-323.

[9] J. Liu and W. Luo, "Routes optimization for PMIPv6 multicast", Internet-Draft, (2012).

[10] T. Chiba, H. Yokota, A. Dutta, D. Chee and H. Schulzinne, "Route optimization for Proxy mobile IPv6 in IML network", 2nd International Conference on Signal Processing and Communication Systems (ICSPCS 2008), (2008), pp. 15-17.

[11] J. C. Zuniga, L. M. Contreras, C. J. Bernardos, S. Jeon and Y. Kim, "Multicast mobility routing optimizations for Proxy Mobile IPv6", RFC 7028, (2013).

[12] D. V. Hugo and H. Asaeda, "Context Transfer Protocol extension for multicast", Internet-Draft, (2013).

[13] J. Loughney, M. Nakhjiri, C. Perkins and R. Koodli, "Context Transfer Protocol”, RFC 4067, (2005).

[14] T. T. Nguyen and C. Bonnet, "Performance optimization of multicast content delivery in a mobile environment based on PMIPv6", Wireless Communications and Networking Conference (WCNC) IEEE, (2013), pp. 1249-1254, doi:10.1109/WCNC.2013.6554743.

[15] A. Karan, C. Filsfils, I. J. Wijnands and B. Decraene, "Multicast Only Fasr Reroute (MoFRR)", RFC 7431, (2015).

[16] T. Schmidt, M. Waehlisch and S. Krishnan, "Base deployment for multicast listener support in Proxy Mobile IPv6 (PMIPv6) domains", RFC 6224, (2011).

[17] R. Vida and L. Costa, "Multicast Listener Discovery Version 2 (MLDv2) for IPv6", RFC 3810, (2004).

[18] M. Gohar, S. I. Choi, S. J. Koh, "Fast handover using multicast handover agents in PMIPv6-based wireless network", The 25th International Conference on Information Networking (ICOIN 2011), (2011).

[19] A. J. Jabir, S. Shamala and Z. Zuriati, "A new strategy for signalling overhead reduction in the proxy mobile IPv6 Protocol”, Am. J. of Applied Sci., vol. 9, iss. 4, (2012), pp. 535-541.

[20] J. Liu and W. Luo, "Routes optimization for PMIPv6 multicast", Internet-Draft, (2012).

[21] T. Chiba, H. Yokota, A. Dutta, D. Chee and H. Schulzinne, "Route optimization for Proxy mobile IPv6 in IML network", 2nd International Conference on Signal Processing and Communication Systems (ICSPCS 2008), (2008).

[22] J. C. Zuniga, L. M. Contreras, C. J. Bernardos, S. Jeon and Y. Kim, "Multicast mobility routing optimizations for Proxy Mobile IPv6", RFC 7028, (2013). 
[23] H. G. Kim, J. M. Kim and H. K. Kim, “A Global Mobility Scheme for Seamless Multicasting in Proxy Mobile IPv6 Networks", Kwangwoon University Industry-Academic Collaboration Foundation, (2012).

[24] T. T. Nguyen and C. Bonnet, "Considerations of IP Multicast for Load Balancing in Proxy Mobile IPv6 Networks", Department of Mobile Communications EURECOM, (2014).

[25] J. Loughney, M. Nakhjiri, C. Perkins and R. Koodli, "Context Transfer Protocol”, RFC 4067, (2005).

[26] D. V. Hugo and H. Asaeda, "Context Transfer Protocol extension for multicast", Internet-Draft, (2013).

[27] T. T. Nguyen and C. Bonnet, "Performance optimization of multicast content delivery in a mobile environment based on PMIPv6", Wireless Communications and Networking Conference (WCNC) IEEE, (2013), pp. 1249-1254. 
International Journal of Future Generation Communication and Networking Vol. 9, No.7, (2016) 